\title{
Modeling estrogen receptor-positive breast cancers in mice: is it the best we can do?
}

\author{
Ioulia Chatzistamou' and Hippokratis Kiaris $2,3,4$ \\ 'Department of Pathology, Microbiology and Immunology, University of South Carolina School of \\ Medicine, Columbia, South Carolina, USA \\ 2Department of Drug Discovery and Biomedical Sciences, University of South Carolina, Columbia, \\ South Carolina, USA \\ 3Peromyscus Genetic Stock Center, Office of Research, University of South Carolina, Columbia, \\ South Carolina, USA \\ ${ }^{4}$ Department of Biochemistry, University of Athens Medical School, Athens, Greece
}

Correspondence should be addressed to $\mathrm{H}$ Kiaris

Email

kiarish@sccp.sc.edu

\begin{abstract}
The continuous supplementation of mice with supraphysiological doses of estrogen for the growth of estrogen receptor-positive breast cancers has been linked to toxicity in the host and perturbation of cancer cells' function that can misguide preclinical studies. Thus, alternative experimental models with circulating levels of estrogens higher than those of mice may represent more suitable hosts to model estrogen receptor-positive breast cancers.
\end{abstract}

\author{
Key Words \\ - estrogen receptor \\ - cancer model \\ - estrogen \\ supplementation \\ - breast cancer
}

Endocrine-Related Cancer (2016) 23, C9-C12
Estrogen receptor (ER)-positive breast cancers account for about two thirds of total breast cancer cases. Of those, about one half is diagnosed in post-menopausal women (http://www.breastcancer.org/symptoms/understand_bc/ statistics). Hormone therapy that consists essentially of either estrogen antagonists or aromatase inhibitors (aromatase is the enzyme that converts androgens to estrogens), remains the treatment of choice for ER-positive breast cancers but despite the positive results it benefits only about two thirds of the patients. Thus, additional research is imperative in order to increase the subset of patients that benefit from the available therapies.

Although several experimental drugs are being continuously developed, only a very small fraction shows promising results in the clinic, which among others also illustrates the limited predictive power of commonly used preclinical models. In parallel with the intensive efforts to improve existing and to develop novel pharmaceuticals, the identification and utilization of better preclinical models that can predict more accurately the outcome of the human disease represents a strategy that usually is being underestimated in the attempts to advance breast cancer treatment choices. For reasons not necessarily relying on purely scientific grounds, the principle effort is conventionally directed towards the development of novel drugs. Implementation though of additional animal models in preclinical testing may have the potential to indirectly improve substantially anticancer management. Such models should predict more accurately the most promising drug candidates increasing the portion of the experimental therapeutics that can make it to the clinic. The commonly experienced failure of several promising drug candidates in the clinic illustrates the limitations of the animal models used most commonly in preclinical testing (Sledge et al. 2014).

The laboratory mouse (Mus musculus) is the animal model of choice for studies in the area of experimental oncology and drug testing because of our deep and

Published by Bioscientifica Ltd. 
extended knowledge of its biology in combination with the wide availability of molecular reagents and methodologies facilitating the manipulation of the mouse genome. However, modeling breast cancer in mice, especially the estrogen-dependent type of the disease, bears several limitations that restrict the preclinical value of this model, rendering it a rather unfortunate choice. The most important limitation of mice in the context of ER-positive breast cancer is related to their low endogenous estrogen levels that mandate estrogen supplementation for efficient breast cancer growth. Thus, while in pre-menopausal women 17ß-estradiol (E2) levels in the plasma reportedly exceed $27 \mathrm{pg} / \mathrm{mL}$ (Folkerd et al. 2014), in adult mice they are limited to levels below $13.7 \mathrm{pg} / \mathrm{mL}$ (Haisenleder et al. 2011). The presence of E2 at sufficient levels, above a certain threshold, is obligatory for the growth of ER-positive breast cancers. This requirement for estrogen finds direct application in the clinical management of the disease, in strategies that aim to inhibit the activity of estrogens and eventually cause inhibition of breast cancer growth. Although primary $\mathrm{ER}(+)$ breast cancers may develop in mice upon genetic manipulations or chemical carcinogenesis, the resulting tumors by being of mouse origin do not represent the most adequate model for drug testing and thus their usage as such is limited.

Supplementation of E2 in mice is commonly applied by implantation of estrogen-releasing pellets that attain at least a 5-fold induction in circulating E2 levels (Offner et al. 2000), which is sufficient and probably excessive to sustain the growth of ER-positive human breast cancers. Two major limitations though are linked to E2 supplementation: The toxicity that is produced in the host from the presence of such supra-physiological E2 levels in the circulation, and the perturbation of cancer cells' function, due to the non-physiological and continuously high, steady levels of E2 that is maintained in the circulation. Both adverse consequences of E2 supplementation, in both the host and the cancer cells, may likely interfere with the tumor's characteristics reducing its value to accurately predict its response against experimental drugs. While utilizing alternative mouse strains may improve the tolerance of experimental mice to estrogen supplementation, the interference with the tumor's physiology persists, rendering the results less relevant to the human disease at which endogenous estrogen levels, under physiological conditions are cycling.

A rather physiological avenue to overcome the limitations associated with the exogenous supplementation of E2 is to utilize alternative animal models that exhibit higher circulating estrogen levels than those observed in mice. It is conceivable that E2 levels above a certain threshold may be sufficient to support the growth of estrogen receptor-positive breast cancers without supplementation of E2. Table 1 shows previously reported baseline E2 levels in the plasma of several small mammals. Although many of the animals described cannot be used as models for breast cancer studies the results described illustrate the high variation that exists with respect to the circulating E2 levels in mammals. As can readily be seen, the laboratory mouse is really a poor choice since E2 levels in all of these animals exceed considerably those of the common laboratory mice and range within levels that occasionally approach and exceed the minimal levels reported in women. Similarly low E2 levels are also present in the rat, another species used to model breast cancer (Ström et al. 2008, Dearth et al. 2014, Rumi et al. 2014). The fact that almost half of the ER-positive breast cancers develop in post-menopausal women, at which E2 levels decrease, strongly supports the hypothesis that ER-positive breast cancers may grow in animals

Table 1 Circulating 17 $\beta$-estradiol levels in various species.

\begin{tabular}{l} 
Taxonomic name \\
\hline Homo sapiens \\
Mus musculus \\
Mus musculus (E2 suppl)b \\
Rattus norvegicus \\
Lagomorpha leporidae \\
Canis mesomelas \\
Canis familiaris \\
Marmota monax \\
Monodelphis domestica \\
Phodopus campbelli \\
Nyctereutes procyonoides
\end{tabular}

\begin{tabular}{l} 
Common name \\
\hline Woman \\
Mouse \\
Mouse \\
Rat \\
Rabbit \\
Black-backed jackal \\
Domestic dog \\
Groundhog \\
Opossum \\
Hamster \\
Raccoon dog
\end{tabular}

\begin{tabular}{c} 
Minimal 17ק-estradiol $(\mathrm{pg} / \mathrm{mL})^{a}$ \\
\hline 27 \\
13.5 \\
150 \\
15 \\
31.8 \\
34 \\
49 \\
34.4 \\
54 \\
54 \\
55
\end{tabular}

\begin{tabular}{l} 
Citation \\
\hline Folkerd et al. (2014) \\
Haisenleder et al. (2011) \\
Offner et al. (2000) \\
Dearth et al. (2014) \\
Batra et al. (1979) \\
Walton and Joly (2003) \\
Fahiminiya et al. (2010) \\
Kwiecinski (1998) \\
Fadem and Harder (1992) \\
Ross (1995) \\
Ward and Wurster-Hill (1990)
\end{tabular}

aE2 levels vary considerably. Lowest circulating levels, in follicular phase, are shown; bValues are attained by utilizing $0.36 \mathrm{mg}$ E2-releasing pellets. 
bearing even moderately higher E2 levels than those of mice. To that end it is conceivable that their circulating estrogen levels will be more similar to those of postmenopausal women.

A major limitation of the data described in Table 1 is that they have been extracted from studies involving different methodologies and analytical reagents, thus precluding a direct and formal side-by-side comparison. Although actual levels may deviate considerably from those shown in Table 1, it still remains highly plausible that other than mice small mammals may exhibit consistently higher E2 levels in the plasma, sufficient to support ER-positive breast cancer growth. In the absence of naturally existing or genetically established immune-incompetent such animals, thymectomy or certain pharmacological interventions could render these animals immunosuppressed, preventing graft rejection and facilitating ER-positive breast cancer growth. Indeed, the use of chemically-induced immunosuppression has been used successfully in mice and rats to grow human cancers and this strategy may likely be effective in other mammals as well (Fingert et al. 1984, Bennett et al. 1985 Goodman et al. 1987).

An additional advantage of such strategy is that by definition will not involve clonal and thus genetically identical animals but rather genetically heterogenous and probably outbred populations. In view of limitations of mice, due to their inbred nature, as models for preclinical testing, using wild type populations of naturally existing species may render them by far superior than mice for preclinical drug evaluation and for other studies at which the heterogeneity of the host must be taken into consideration.

Identification of small mammals with high endogenous E2, sufficient to support ER-positive breast cancer growth may have the potential to provide informative preclinical models, devoid of the major limitations linked to the E2 supplementation that is mandatory for experimentation with laboratory mice. The high variability in circulating E2 levels mammals, in combination with the fact that likely mice possess E2 levels in the lower range, supports this notion. Thus, instead of improving existing models, seeking novel ones on the basis of their intrinsic characteristics and endocrine profile, may provide a valid strategy for exploring new avenues in screening of novel breast cancer therapeutics. It is probably the right time to reconsider how experimental drugs are being tested before they enter in the clinical tests, and whether mice are the best choice from what can be presently available.
Declaration of interest

The authors declare that there is no conflict of interest that could be perceived as prejudicing the impartiality of this commentary.

\section{Funding}

This work did not receive any specific grant from any funding agency in the public, commercial, or not-for-profit sector.

\section{Acknowledgement}

Work on breast cancer in the authors' lab is supported by a pilot grant 5P30GM103336-02 from NIH (to HK) and by an ASPIRE-I grant 18060-1641667 from USC Office of Research (to IC).

\section{References}

Batra S, Owman C, Sjöberg NO \& Thorbert G 1979 Relationship between plasma and uterine oestradiol in pseudopregnant rabbits. Journal of Reproduction and Fertility 56 1-5. (doi:10.1530/ jrf.0.0560001)

Bennett JA, Pilon VA, Briggs DR \& McKneally MF 1985 Evaluation of cyclosporine-treated mice as hosts for growing and testing the chemosensitivity of first-transplant-generation human tumor xenografts implanted under the kidney capsule. Journal of the National Cancer Institute 75 925-936.

Dearth RK, Hiney JK, Srivastava VK, Hamilton AM \& Dees WL 2014 Prepubertal exposure to elevated manganese results in estradiol regulated mammary gland ductal differentiation and hyperplasia in female rats. Experimental Biology and Medicine 239 871-882. (doi:10.1177/1535370214531865)

Fadem BH \& Harder JD 1992 Estrogen in peripheral plasma during postnatal development in gray short-tailed opossums. Physiology and Behavior 52 613-616. (doi:10.1016/0031-9384(92)90356-7)

Fahiminiya S, Reynaud K, Labas V, Batard S, Chastant-Maillard S \& Gérard N 2010 Steroid hormones content and proteomic analysis of canine follicular fluid during the preovulatory period. Reproductive Biology and Endocrinology 8 132. (doi:10.1186/1477-7827-8-132)

Fingert HJ, Treiman A \& Pardee AB 1984 Transplantation of human or rodent tumors into cyclosporine-treated mice: a feasible model for studies of tumor biology and chemotherapy. PNAS 81 7927-7931. (doi:10.1073/pnas.81.24.7927)

Folkerd EJ, Lønning PE \& Dowsett M 2014 Interpreting plasma estrogen levels in breast cancer: caution needed. Journal of Clinical Oncology 32 1396-1400. (doi:10.1200/JCO.2013.53.9411)

Goodman MM, McCullough JL, Biren CA \& Barr RJ 1987 A model of human melanoma in cyclosporine-immunosuppressed rats. Journal of Investigative Dermatology 88 141-144. (doi:10.1111/1523-1747. ep12525289)

Haisenleder DJ, Schoenfelder AH, Marcinko ES, Geddis LM \& Marshall JC 2011 Estimation of estradiol in mouse serum samples: evaluation of commercial estradiol immunoassays. Endocrinology 152 4443-4447. (doi:10.1210/en.2011-1501)

Kwiecinski GG 1998 Mammalian species: Marmota monax. American Society of Mammalogists 591 1-8. (doi:10.2307/3504364)

Offner H, Adlard K, Zamora A \& Vandenbark AA 2000 Estrogen potentiates treatment with T-cell receptor protein of female mice with experimental encephalomyelitis. Journal of Clinical Investigation 105 1465-1472. (doi:10.1172/JCI9213)

Ross PD 1995 Mammalian species: Phodopus campbelli (hamster). American Society of Mammalogists 503 1-7. (doi:10.2307/3504253)

Rumi MA, Dhakal P, Kubota K, Chakraborty D, Lei T, Larson MA, Wolfe MW, Roby KF, Vivian JL \& Soares MJ 2014 Generation of 
Esr1-knockout rats using zinc finger nuclease-mediated genome editing. Endocrinology 155 1991-1999. (doi:10.1210/en.2013-2150)

Sledge GW, Mamounas EP, Hortobagyi GN, Burstein HJ, Goodwin PJ \& Wolff AC 2014 Past, present, and future challenges in breast cancer treatment. Journal of Clinical Oncology 32 1979-1986. (doi:10.1200/ JCO.2014.55.4139)

Ström JO, Theodorsson A \& Theodorsson E 2008 Substantial discrepancies in 17beta-oestradiol concentrations obtained with three different commercial direct radioimmunoassay kits in rat sera. Scandinavian Journal of Clinical and Laboratory Investigation $\mathbf{6 8}$ 806-813. (doi:10.1080/00365510802254638)

Walton LR \& Joly DO 2003 Mammalian species: Canis mesomelas (black-backed jackal). American Society of Mammalogists 715 1-9.

Ward OG \& Wurster-Hill DH 1990 Mammalian species: Nyctereutes procyonoides (raccoon dog). American Society of Mammalogists $\mathbf{3 5 8}$ 1-5. (doi:10.2307/3504213)

Received in final form 6 September 2016

Accepted 9 September 2016

Accepted Preprint published online 12 September 2016
Published by Bioscientifica Ltd. 